INPLASY

PROTOCOL

To cite: Li et al. Effectiveness of acupuncture for irritable bowel syndrome: Protocol for a scoping review of systematic reviews and meta-analyses. Inplasy protocol 202210117. doi:

10.37766/inplasy2022.1.0117

Received: 24 January 2022

Published: 24 January 2022

Corresponding author:

Yachen Li

ljlijia@163.com

Author Affiliation:

Hubei University of Chinese

Medicine.

Support: NSFC No: 81804165 and 81973931.

Review Stage at time of this submission: Preliminary

searches.

Conflicts of interest:

None declared.

\section{Effectiveness of acupuncture for irritable bowel syndrome: Protocol for a scoping review of systematic reviews and meta-analyses}

\author{
Li, Y1; Peng, S2; Liang, F3; Liu, S4; Li, J5.
}

Review question / Objective: To provide a scoping review of current systematic reviews (SRs)/meta-analyses (MAs) on the efficacy and safety of acupuncture for irritable bowel syndrome (IBS), and to assess the methodological quality and the strength of evidence of the included SRs/MAs.

Condition being studied: Irritable bowel syndrome (IBS), a common functional bowel disorder, is characterized by recurrent abdominal pain and discomfort that include defecation or a change in bowel habits. According to the statistics, IBS affects about $11 \%$ of the world's population. Yet it significantly affects the quality of life and work productivity of patients, and contributes to economic loss, imposing a great socioeconomic burden. Acupuncture is used and frequently advocated for the treatment of IBS. This scoping review aims to summarize the available evidence from current SRs and MAs for the efficacy and safety of acupuncture for IBS.

INPLASY registration number: This protocol was registered with the International Platform of Registered Systematic Review and Meta-Analysis Protocols (INPLASY) on 24 January 2022 and was last updated on 24 January 2022 (registration number INPLASY202210117).

\section{INTRODUCTION}

Review question / Objective: To provide a scoping review of current systematic reviews (SRs)/meta-analyses (MAs) on the efficacy and safety of acupuncture for irritable bowel syndrome (IBS), and to assess the methodological quality and the strength of evidence of the included SRs/ MAs.

Rationale: With the deepening and progress of clinical evidence-based, many clinical studies of acupuncture intervention in IBS have emerged at home and abroad, and relevant SRs and MAs have been 
published continuously. However, only high-quality SRs and MAs can provide doctors and clinical research investigators with reliable information and research direction for future studies. Low-quality studies are not only easy to mislead decision-makers but also seriously reduce the value and authenticity of research results. Therefore, there is an urgent need to synthesize evidence on the effectiveness and safety of acupuncture in the treatment of irritable bowel syndrome from SRs and MAs.

Condition being studied: Irritable bowel syndrome (IBS), a common functional bowel disorder, is characterized by recurrent abdominal pain and discomfort that include defecation or a change in bowel habits. According to the statistics, IBS affects about $11 \%$ of the world's population. Yet it significantly affects the quality of life and work productivity of patients, and contributes to economic loss, imposing a great socioeconomic burden. Acupuncture is used and frequently advocated for the treatment of IBS. This scoping review aims to summarize the available evidence from current SRs and MAs for the efficacy and safety of acupuncture for IBS.

\section{METHODS}

Search strategy: We will design a systematic and careful retrieval scheme, and search PubMed, Embase, Cochrane Database, China National Knowledge Infrastructure (CNKI), China Science and Technology Journal Database (VIP), China Biology Medicine disc (CBMdisc), and Wanfang Database since the establishment of the database. Search terms include "irritable bowel syndrome", "acupuncture", "meta-analysis" and their synonyms.

Participant or population: We will include patients diagnosed with irritable bowel syndrome.

Intervention: Acupuncture, electroacupuncture, auricular acupuncture, etc., or any combination of the above.
Comparator: Conventional drugs, sham acupuncture, placebo, and no treatment will be permissible as the comparators.

Study designs to be included: Systematic reviews and meta-analyses.

Eligibility criteria: Study inclusion criteria: (1) qualified literature of systematic review and meta-analysis of all relevant acupuncture interventions in IBS. (2) Include at least a kind of acupuncture therapy, such as acupuncture, electroacupuncture, auricular acupuncture, etc.(3) Outcomes included global IBS symptoms, quality of life scores, IBS symptom scores, etc.Study exclusion criteria:(1) documents with incomplete article content or information that cannot be extracted.(2) documents discussed in narrative and methodology, systematic evaluation related to etiology, pathogenesis, and diagnostics, planning book of systematic evaluation, summary of systematic review/meta-analysis, or translated documents.

Information sources: Seven key electronic databases, including PubMed, Embase, Cochrane Database, China National Knowledge Infrastructure (CNKI), China Science and Technology Journal Database (VIP), China Biology Medicine disc (CBMdisc), and Wanfang Database will be searched since the establishment of the database. There will be no limits on languages.

Main outcome(s): The total effective rates, the cure rate, the overall IBS symptom scores, or global quality of life scores.

\section{Additional outcome(s): None.}

Data management: The information collection form of SRs and MAs literature quality evaluation of acupuncture treatment of irritable bowel will be established, including publication time, the first author, nationality, sample size, intervention measures of the test group and controlled group, quality assessment tools used, main indicators, main conclusions, etc. 
Quality assessment / Risk of bias analysis: Two researchers (LSZ and LFY) will use the AMSTAR2 methodological quality assessment scale and PRISMA report quality assessment scale. The quality evaluation of the methodology included in the table is completed independently. If the evaluation is inconsistent, a consensus is reached through discussion.

Strategy of data synthesis: The key elements of the review available include the number of RCT included in SRs/ MAs, total sample size, intervention, control, and outcome measurements. The data extracted from the study is presented in the form of tables and pictures to facilitate our summary. The results will be simply organized in tabular format, and narrative descriptions will be used where further analysis is required.

Subgroup analysis: As scoping review research, there will be no plan for analyzing subgroup data.

Sensitivity analysis: As scoping review research, there will be no plan to perform the sensitivity analysis of data.

Language: No restrictions will be applied to language.

Country(ies) involved: China.

Keywords: Acupuncture, irritable bowel syndrome, scoping review, systematic reviews and meta-analyses.

Dissemination plans: The findings will be published in peer-reviewed journals and/or presented at scientific conferences.

Contributions of each author:

Author 1 - Yachen Li.

Email: 361113080@qq.com

Author 2 - Sike Peng.

Author 3 - Fangyuan Liang.

Author 4 - Suzhen Liu.

Author 5 - Jia Li.

Email: Ijlijia@163.com 